\title{
Lepton-number violation in $B_{s}$ meson decays induced by an on-shell Majorana neutrino
}

\author{
Jhovanny Mejía-Guisao, ${ }^{1, *}$ Diego Milanés, ${ }^{2, \dagger}$ Néstor Quintero, ${ }^{3, *}$ and José D. Ruiz-Álvarez, ${ }^{4, \S}$ \\ ${ }^{1}$ Departamento de Física, Centro de Investigación y de Estudios Avanzados del IPN, \\ Apartado Postal 14-740, 07000 Ciudad de México, México \\ ${ }^{2}$ Departamento de Física, Universidad Nacional de Colombia, Código Postal 11001, Bogotá, Colombia \\ ${ }^{3}$ Facultad de Ciencias Básicas, Universidad Santiago de Cali, Campus Pampalinda, \\ Calle 5 No. 62-00, Código Postal 76001, Santiago de Cali, Colombia \\ ${ }^{4}$ Departamento de Física, Universidad de Los Andes, Código Postal 111711, Bogotá, Colombia
}

(Received 10 February 2018; published 16 April 2018)

\begin{abstract}
Lepton-number violation can be induced by the exchange of an on-shell Majorana neutrino $N$ in semileptonic $|\Delta L|=2$ decays of the $B_{s}$ meson, $B_{s}^{0} \rightarrow P^{-} \pi^{-} \mu^{+} \mu^{+}$with $P=K, D_{s}$. We investigate the production of such a heavy sterile neutrino through these four-body $\mu^{+} \mu^{+}$channels and explore the sensitivity that can be reached at the LHCb and CMS experiments. For heavy neutrino lifetimes of $\tau_{N}=[1,100,1000] \mathrm{ps}$ and integrated luminosities collected of 10 and $50 \mathrm{fb}^{-1}$ at the LHCb and 30, 300, and $3000 \mathrm{fb}^{-1}$ at the CMS, we find a significant sensitivity on branching fractions of the orders $\operatorname{BR}\left(B_{s}^{0} \rightarrow K^{-} \pi^{-} \mu^{+} \mu^{+}\right) \lesssim \mathcal{O}\left(10^{-9}-10^{-8}\right)$ and $\operatorname{BR}\left(B_{s}^{0} \rightarrow D_{s}^{-} \pi^{-} \mu^{+} \mu^{+}\right) \lesssim \mathcal{O}\left(10^{-8}-10^{-7}\right)$. In the kinematically allowed mass ranges of $m_{N} \in[0.25,4.77] \mathrm{GeV}$ and $m_{N} \in[0.25,3.29] \mathrm{GeV}$, respectively, we exclude regions on the parameter space $\left(m_{N},\left|V_{\mu N}\right|^{2}\right)$ associated with the heavy neutrino, which could slightly improve the limits from $B^{-} \rightarrow \pi^{+} \mu^{-} \mu^{-}$(LHCb).
\end{abstract}

DOI: 10.1103/PhysRevD.97.075018

\section{INTRODUCTION}

To discriminate if the light neutrinos are Majorana or Dirac fermions (i.e., if neutrinos are their own antiparticles or not) is one of the most important puzzles in the Standard Model (SM) [1]. To date, it is already well confirmed by a diversity of neutrino oscillation experiments (solar, atmospheric, reactors, and accelerators) [2] that light neutrinos are massive particles; however, the responsible underlying mechanism remains unknown, and different new physics (NP) scenarios beyond the SM predict the neutrinos to be Dirac or Majorana massive fermions [3]. If neutrinos are Dirac massive particles, the total lepton number $L$ is a conserved quantity in the nature, while if neutrinos turn out to be Majorana massive particles, $L$ will be not longer conserved and will be violated [1]. The most remarkable searches of lepton-number violating (LNV) signals are by

\footnotetext{
*jmejia@ fis.cinvestav.mx

diego.milanes@cern.ch

*nestor.quintero01@usc.edu.co

§jose.ruiz@cern.ch
}

Published by the American Physical Society under the terms of the Creative Commons Attribution 4.0 International license. Further distribution of this work must maintain attribution to the author(s) and the published article's title, journal citation, and DOI. Funded by SCOAP. looking for processes with $|\Delta L|=2$, in which the possible existence of Majorana neutrinos can be tested [1].

The smoking-gun LNV signal is the neutrinoless double$\beta(0 \nu \beta \beta)$ decay [4-6]. Searches of this rare nuclear transition have been pursued for several decades by different experiments, and up to now no positive signal has been observed [4-6]. Currently, the best limits on their half-lives have been obtained from the nuclei ${ }^{76} \mathrm{Ge}[7]$ and ${ }^{136} \mathrm{Xe}[8,9]$. Aside from the $0 \nu \beta \beta$ decay, low-energy studies of rare semileptonic processes in $|\Delta L|=2$ decays of pseudoscalar mesons $\left(K, D, D_{s}, B, B_{c}\right)$ and the $\tau$ lepton have been considered as complementary and alternative evidence to prove the Majorana nature of neutrinos [10-37]. Since these $|\Delta L|=2$ decays can be produced (and enhanced) via an intermediate on-shell Majorana neutrino $N$ with a mass in the range $\sim[0.1,5.0] \mathrm{GeV}$, the phenomenology associated with such a heavy neutrino has been actively studied [10,13-37]. From the experimental side, upper limits on the branching fractions of various LNV processes have been set by different experiments such NA48/2, BABAR, Belle, LHCb, and E791 [38-46]. See also the Particle Data Group [2].

Focusing on the $b$-quark sector, recent attention has been paid to the four-body $|\Delta L|=2$ decays of $B$ and $B_{c}$ mesons: $\bar{B}^{0} \rightarrow D^{+} \pi^{+} \mu^{-} \mu^{-}, \quad B^{-} \rightarrow D^{0} \pi^{+} \mu^{-} \mu^{-} \quad[23,25,34,35]$, and $B_{c}^{-} \rightarrow J / \psi \pi^{+} \mu^{-} \mu^{-}$[20,21]. In addition, the $|\Delta L|=2$ decays of $\Lambda_{b}$ baryon have been explored as well [47]. 
As a salient feature, these decay channels are not highly suppressed by Cabbibo-Kobayashi-Maskawa (CKM) factors, and their experimental search is within reach of sensitivity of the LHCb and Belle II $[20,21,35]$. So far, the $\mathrm{LHCb}$ has reported the upper limit $\operatorname{BR}\left(B^{-} \rightarrow\right.$ $\left.D^{0} \pi^{+} \mu^{-} \mu^{-}\right)<1.5 \times 10^{-6}$ [41], and improvements are expected in Run 2 and the future upgrade Run 3. On the other hand, the same quark level LNV transition that generates these four-body $|\Delta L|=2$ channels can also produce $|\Delta L|=2$ decays in the $B_{s}$ meson, and their signals may be detected at the LHC, which offers an excellent environment for the $B_{s}$ physics.

In this work, we will explore the LNV decay channels of the $B_{s}$ meson, $B_{s}^{0} \rightarrow P^{-} \pi^{-} \mu^{+} \mu^{+}$with $P=K, D_{s}$, via an intermediate $\mathrm{GeV}$-scale on-shell Majorana neutrino $N$. To our knowledge, these $|\Delta L|=2$ decays have not been investigated before from a theoretical nor from an experimental point of view. We will work in a simplified approach in which one heavy neutrino $N$ mixes with one flavor of SM lepton $\ell$ and its interactions are completely determined by the mixing angle $V_{\ell N}$ [10]. Since $0 \nu \beta \beta$ decay puts stringent limits on the electron-heavy neutrino mixing $\left|V_{e N}\right|^{2} \lesssim 10^{-8}$ [48], we will focus our attention on the above four-body $\mu^{+} \mu^{+}$modes and explore their expected sensitivities at the $\mathrm{LHCb}$ and CMS experiments. We will show that their experimental search allows us to scan the parameter space $\left(m_{N},\left|V_{\mu N}\right|^{2}\right)$ of the heavy neutrino sector, therefore an additional test of the existence of Majorana neutrinos.

Let us mention that the presence of a heavy neutrino with a mass of few $\mathrm{GeV}[\sim \mathcal{O}(1) \mathrm{GeV}]$ provides a realistic and falsifiable scenario for a common explanation of the baryon asymmetry of the Universe via leptogenesis [49-52] and the generation of neutrino masses via the $\mathrm{GeV}$-scale seesaw model $[53,54]$. This gives us further motivation to study $|\Delta L|=2$ decays of the $B_{s}$ meson under consideration.

This work is organized as follows. In Sec. II, we study the $|\Delta L|=2$ decays of the $B_{s}$ meson. The expected experimental sensitivities for these channels at the LHCb and CMS is discussed in Sec. III. Based on the results of Sec. III, we discuss the bounds on the parameter space $\left(m_{N},\left|V_{\mu N}\right|^{2}\right)$ of the heavy neutrino that can be achieved. Our conclusions are given in Sec. V.

\section{LNV DECAYS OF $B_{s}$ MESON}

In this section, we study LNV signals in the $|\Delta L|=2$ decays of the $B_{s}$ meson $B_{s}^{0} \rightarrow P^{-} \pi^{-} \mu^{+} \mu^{+}$, with $P=K, D_{s}$ denoting a final-state pseudoscalar meson. These processes can occur via intermediate on-shell Majorana neutrino $N$ through the semileptonic decay $B_{s}^{0} \rightarrow P^{-} \mu^{+} N$ followed by the subsequent decay $N \rightarrow \mu^{+} \pi^{-}$, with a kinematically allowed mass in the ranges

$$
\begin{aligned}
& B_{s}^{0} \rightarrow K^{-} \pi^{-} \mu^{+} \mu^{+}: m_{N} \in[0.25,4.77] \mathrm{GeV}, \\
& B_{s}^{0} \rightarrow D_{s}^{-} \pi^{-} \mu^{+} \mu^{+}: m_{N} \in[0.25,3.29] \mathrm{GeV} .
\end{aligned}
$$

The $B_{s}^{0} \rightarrow P^{-} \pi^{-} \mu^{+} \mu^{+}$decays are then split into two subprocesses, and the corresponding branching fraction can be written in the factorized form

$$
\begin{aligned}
\operatorname{BR}\left(B_{s}^{0} \rightarrow P^{-} \pi^{-} \mu^{+} \mu^{+}\right)= & \operatorname{BR}\left(B_{s}^{0} \rightarrow P^{-} \mu^{+} N\right) \\
& \times \Gamma\left(N \rightarrow \mu^{+} \pi^{-}\right) \tau_{N} / \hbar,
\end{aligned}
$$

with $\tau_{N}$ as the lifetime of the Majorana neutrino. The branching ratio of $B_{s}^{0} \rightarrow P^{-} \mu^{+} N$ is given by the expression [34]

$$
\begin{aligned}
& \operatorname{BR}\left(B_{s}^{0} \rightarrow P^{-} \mu^{+} N\right) \\
& \quad=\left|V_{\mu N}\right|^{2} \int d t \frac{d \overline{\mathrm{BR}}\left(B_{s}^{0} \rightarrow P^{-} \mu^{+} N\right)}{d t},
\end{aligned}
$$

where

$$
\begin{aligned}
& \frac{d \overline{\mathrm{BR}}\left(B_{s}^{0} \rightarrow P^{-} \mu^{+} N\right)}{d t} \\
& =\frac{G_{F}^{2} \tau_{B_{s}}}{384 \pi^{3} m_{B_{s}}^{3} \hbar}\left|V_{q b}^{\mathrm{CKM}}\right|^{2} \frac{\left[\lambda\left(m_{\mu}^{2}, m_{N}^{2}, t\right) \lambda\left(m_{B_{s}}^{2}, m_{P}^{2}, t\right)\right]^{1 / 2}}{t^{3}} \\
& \quad \times\left(\left[F_{+}^{B_{s} P}(t)\right]^{2} C_{+}(t)+\left[F_{0}^{B_{s} P}(t)\right]^{2} C_{0}(t)\right)
\end{aligned}
$$

is the so-called differential canonical branching ratio [34], where $G_{F}$ is the Fermi constant; $V_{q b}^{\mathrm{CKM}}$ denotes the CKM matrix element involved (with $q=u, c$ for $\left.P=K, D_{s}\right)^{1}$; and $F_{+}^{B_{s} P}(t)$ and $F_{0}^{B_{s} P}(t)$ are the vector and scalar form factors for the $B_{s} \rightarrow P$ transition, respectively, which are evaluated at the square of the transferred momentum $t=\left(p_{B_{s}}-p_{P}\right)^{2}$. The usual kinematic Källen function is denoted by $\lambda(x, y, z)=x^{2}+y^{2}+z^{2}-2(x y+x z+y z)$. The coefficients $C_{+}(t)$ and $C_{0}(t)$ in (3) are defined as

$C_{+}(t)=\lambda\left(m_{B_{s}}^{2}, m_{P}^{2}, t\right)\left[2 t^{2}+t\left(m_{\mu}^{2}+m_{N}^{2}\right)+\left(m_{\mu}^{2}-m_{N}^{2}\right)^{2}\right]$,

$C_{0}(t)=3\left(m_{B_{s}}^{2}-m_{P}^{2}\right)\left[m_{\mu}^{2}\left(t+2 m_{N}^{2}-m_{\mu}^{2}\right)+m_{N}^{2}\left(t-m_{N}^{2}\right)\right]$,

respectively. The total branching fraction is then obtained by integrating the differential canonical branching ratio over the full $t$ region $\left[\left(m_{\mu}+m_{N}\right)^{2},\left(m_{B_{s}}-m_{P}\right)^{2}\right]$.

As mentioned at the Introduction, the coupling of the heavy neutrino (sterile) $N$ to the charged current of lepton flavor $\mu$ is characterized by the quantity $V_{\mu N}$ [10]. Without referring to any NP scenario, we will treat $m_{N}$ and $V_{\mu N}$ as unknown phenomenological parameters that can be

\footnotetext{
${ }^{1}$ We will use the central values $\left|V_{u b}^{\mathrm{CKM}}\right|=4.09 \times 10^{-3}$ and $\left|V_{c b}^{\mathrm{CKM}}\right|=40.5 \times 10^{-3}[2]$.
} 
constrained (set) from the experimental nonobservation (observation) of $|\Delta L|=2$ processes [10,15,20].

On the other hand, the decay width of $N \rightarrow \mu^{+} \pi^{-}$is given by the expression [10]

$$
\Gamma\left(N \rightarrow \mu^{+} \pi^{-}\right)=\left|V_{\mu N}\right|^{2} \bar{\Gamma}\left(N \rightarrow \mu^{-} \pi^{+}\right),
$$

with

$$
\begin{aligned}
\bar{\Gamma}\left(N \rightarrow \mu^{+} \pi^{-}\right)= & \frac{G_{F}^{2}}{16 \pi}\left|V_{u d}^{\mathrm{CKM}}\right|^{2} f_{\pi}^{2} m_{N} \sqrt{\lambda\left(m_{N}^{2}, m_{\mu}^{2}, m_{\pi}^{2}\right)} \\
& \times\left[\left(1-\frac{m_{\mu}^{2}}{m_{N}^{2}}\right)^{2}-\frac{m_{\pi}^{2}}{m_{N}^{2}}\left(1+\frac{m_{\mu}^{2}}{m_{N}^{2}}\right)\right],
\end{aligned}
$$

where $\left|V_{u d}^{\mathrm{CKM}}\right|=0.97417$ [2] and $f_{\pi}=130.2(1.7) \mathrm{MeV}$ is the pion decay constant [55].

The lifetime of the Majorana neutrino $\tau_{N}=\hbar / \Gamma_{N}$ in Eq. (1) can be obtained by summing over all accessible final states that can be opened at the mass $m_{N}$ [10]. However, in further analysis (Secs. III and IV), we will leave it as a phenomenological parameter accessible to the $\mathrm{LHCb}$ and CMS experiments.

\section{A. Form factors $B_{s} \rightarrow P\left(P=K, D_{s}\right)$}

For the form factors associated with the $B_{s} \rightarrow P$ transition, we will use the theoretical predictions provided by the lattice QCD approach [56,57].

The form factors $F_{+}^{B_{s} D_{s}}$ and $F_{0}^{B_{s} D_{s}}$ can be represented by the $z$ expansion through a modification of the BourrelyCaprini-Lellouch (BCL) parametrization [56],

$F_{+}^{B_{s} P}(t)=\frac{1}{\left(1-t / M_{+}^{2}\right)} \sum_{n=0}^{J-1} b_{n}^{+}\left[z(t)^{n}-(-1)^{n-J} \frac{n}{J} z(t)^{J}\right]$,

$F_{0}^{B_{s} P}(t)=\frac{1}{\left(1-t / M_{0}^{2}\right)} \sum_{n=0}^{J-1} b_{n}^{0} z(t)^{n}$

respectively, where the $z(t)$ function is defined as

$$
z(t)=\frac{\sqrt{t_{+}-t}-\sqrt{t_{+}-t_{0}}}{\sqrt{t_{+}-t}+\sqrt{t_{+}-t_{0}}} .
$$

In Table I, we show the respective coefficients of the $z$ expansion in Eqs. (8) and (9) for $J=3$ as well as additional parameters: pole masses $M_{+(0)}$ and $t_{+(0)}$ [56]. The masses of particles involved are taken from the Particle Data Group [2].

In Ref. [57], the form factors for the $B_{s} \rightarrow K$ transition are parametrized in a modified BCL form,
TABLE I. Coefficients $\left(b_{0}^{+}, b_{1}^{+}, b_{2}^{+}\right)$and $\left(b_{0}^{0}, b_{1}^{0}, b_{2}^{0}\right)$ of the $z$ expansion in Eqs. (8) and (9), pole masses $M_{+(0)}$ and $t_{+(0)}$.

\begin{tabular}{lc}
\hline \hline Parameter & $B_{s} \rightarrow D_{s}[56]$ \\
\hline$M_{+}(\mathrm{GeV})$ & 6.330 \\
$M_{0}(\mathrm{GeV})$ & 6.420 \\
$t_{+}\left(\mathrm{GeV}^{2}\right)$ & $\left(m_{B_{s}}+m_{D_{s}}\right)^{2}$ \\
$t_{0}\left(\mathrm{GeV}^{2}\right)$ & $\left(m_{B_{s}}-m_{D_{s}}\right)^{2}$ \\
$b_{0}^{+}$ & 0.858 \\
$b_{1}^{+}$ & -3.38 \\
$b_{2}^{+}$ & 0.6 \\
$b_{0}^{0}$ & 0.658 \\
$b_{1}^{0}$ & -0.10 \\
$b_{2}^{0}$ & 1.3 \\
\hline \hline
\end{tabular}

TABLE II. Coefficients $\left(a_{0}^{+}, a_{1}^{+}, a_{2}^{+}\right)$and $\left(a_{1}^{0}, a_{2}^{0}, a_{3}^{0}\right)$ of the $z$ expansion in Eqs. (11) and (12), pole masses $M_{+(0)}$ and $t_{+(0)}$.

\begin{tabular}{lc}
\hline \hline Parameter & $B_{s} \rightarrow K[57]$ \\
\hline$M_{+}(\mathrm{GeV})$ & 5.3252 \\
$M_{0}(\mathrm{GeV})$ & 5.6794 \\
$t_{+}\left(\mathrm{GeV}^{2}\right)$ & $\left(m_{B_{s}}+m_{K}\right)^{2}$ \\
$t_{0}\left(\mathrm{GeV}^{2}\right)$ & $\left(m_{B_{s}}+m_{K}\right)\left(\sqrt{m_{B_{s}}}-\sqrt{m_{K}}\right)^{2}$ \\
$a_{0}^{+}$ & 0.368 \\
$a_{1}^{+}$ & -0.750 \\
$a_{2}^{+}$ & 2.72 \\
$a_{1}^{0}$ & 0.315 \\
$a_{2}^{0}$ & 0.945 \\
$a_{3}^{0}$ & 2.391 \\
\hline \hline
\end{tabular}

$F_{+}^{B_{s} K}(t)=\frac{1}{\left(1-t / M_{+}^{2}\right)} \sum_{n=0}^{2} a_{n}^{+}\left[z(t)^{n}-(-1)^{n-3} \frac{n}{3} z(t)^{3}\right]$,

$$
\begin{aligned}
F_{0}^{B_{s} K}(t)= & \sum_{n=1}^{3} a_{n}^{0}\left(z(t)^{n}-z(0)^{n}\right) \\
& +\sum_{n=0}^{2} a_{n}^{+}\left[z(0)^{n}-(-1)^{n-3} \frac{n}{3} z(0)^{3}\right],
\end{aligned}
$$

where the corresponding expansion coefficients $\left(a_{0}^{+}, a_{1}^{+}, a_{2}^{+}\right)$ and $\left(a_{1}^{0}, a_{2}^{0}, a_{3}^{0}\right)$, pole masses $M_{+(0)}$ and $t_{+(0)}$, are displayed in Table II.

\section{EXPECTED EXPERIMENTAL SENSITIVITY AT THE LHC}

Now, let us provide an estimation of the expected number of events at the LHC, namely, LHCb and CMS experiments, for the $|\Delta L|=2$ channels of the $B_{s}$ meson, $B_{s}^{0} \rightarrow P^{-} \pi^{-} \mu^{+} \mu^{+}$(with $P=K, D_{s}$ ), discussed above. 


\section{A. LHCb experiment}

The number of expected events in the LHCb experiment has the form

$$
\begin{aligned}
N_{\exp }^{\mathrm{LHCb}}= & \sigma\left(p p \rightarrow H_{b} X\right)_{\mathrm{acc}} f\left(b \rightarrow B_{s}\right) \mathrm{BR}\left(B_{s} \rightarrow \Delta L=2\right) \\
& \times \epsilon_{D}^{\mathrm{LHCb}}\left(B_{s} \rightarrow \Delta L=2\right) P_{N}^{\mathrm{LHCb}} \mathcal{L}_{\text {int }}^{\mathrm{LHCb}},
\end{aligned}
$$

where $\sigma\left(p p \rightarrow H_{b} X\right)_{\text {acc }}$ is the production cross section of $b$ hadrons inside the LHCb geometrical acceptance; $f\left(b \rightarrow B_{s}\right)$ is the hadronization factor of a $b$ quark to the $B_{s}$ meson; $\mathcal{L}_{\text {int }}^{\mathrm{LHCb}}$ is the integrated luminosity; $\operatorname{BR}\left(B_{s} \rightarrow \Delta L=2\right)$ corresponds to the branching fraction of the given LNV process; and $\epsilon_{D}^{\mathrm{LHCb}}\left(B_{s} \rightarrow \Delta L=2\right)$ is its detection efficiency of the LHCb detector involving reconstruction, selection, trigger, particle misidentification, and detection efficiencies. Most of the on-shell neutrinos produced in the decays $B_{s}^{0} \rightarrow\left(K^{-}, D_{s}^{-}\right) \mu^{+} N$ are expected to live a long enough time to travel through the detector and decay $\left(N \rightarrow \mu^{+} \pi^{-}\right)$far from the interaction region. This effect is given by the $P_{N}^{\mathrm{LHCb}}$ factor (acceptance factor), which accounts for the probability of the on-shell neutrino $N$ decay products to be inside the LHCb detector acceptance [30]. The reconstruction efficiency will depend on this acceptance factor as well.

The production cross section has been measured to be $\sigma\left(p p \rightarrow H_{b} X\right)_{\text {acc }}=(75.3 \pm 5.4 \pm 13.0) \mu \mathrm{b}$ inside the LHCb acceptance [58]. The world average for the hadronization factor is taken to be $f\left(b \rightarrow B_{s}\right)=0.103 \pm 0.005$ [59]. The proper computation of the detection efficiency requires fully simulated Monte Carlo samples of the exclusive decay, reconstructed in the same way as real $\mathrm{LHCb}$ data. Here, we perform a rough estimation of the detection efficiency, based on extrapolation of detection efficiencies already reported by $\mathrm{LHCb}$ experiment of similar final states.

The LHCb Collaboration has measured the detection efficiency of the $B_{s}^{0} \rightarrow \phi\left(K^{+} K^{-}\right) \mu^{+} \mu^{-}$decay mode to be $1.1 \%$ [60]. This measurement includes trigger, tracking, reconstruction, particle identification, and selection efficiency. Given the content of final-state charged tracks, we can consider the $B_{s}^{0} \rightarrow K^{-} \pi^{-} \mu^{+} \mu^{+}$to be the same as for the $B_{s} \rightarrow \phi\left(K^{+} K^{-}\right) \mu^{+} \mu^{-}$decay. Regarding the $B_{s}^{0} \rightarrow$ $D_{s}^{-} \pi^{-} \mu^{+} \mu^{+}$decay, a golden mode to reconstruct the $D_{s}^{+}$meson hadronically is $D_{s}^{+} \rightarrow K^{+} K^{-} \pi^{+}$, where $\operatorname{BR}\left(D_{s}^{+} \rightarrow K^{+} K^{-} \pi^{+}\right)=(5.45 \pm 0.17) \times 10^{-2}$ [2]. In this situation, there will be two additional charged tracks in the final state; thus, we can multiply previous efficiency by 0.9 for each additional charged track, the approximated single track reconstruction efficiency at LHCb. Finally, in Ref. [61], reconstruction efficiencies for hypothetical long-lived particles inside the LHCb acceptance are given. Here, we can observe that a maximum variation of about $25 \%$ is measured in the efficiencies of particles living in the [5-50] ps range, with masses up to $200 \mathrm{GeV}$; however, in our case, long-lived particles can only be produced on shell, therefore with masses around few GeV. Thus, to account for this effect, we will just add a $25 \%$ relative uncertainty to our efficiency prediction, obtaining finally

$$
\begin{aligned}
& \epsilon_{D}^{\mathrm{LHCb}}\left(B_{s} \rightarrow K^{-} \pi^{-} \mu^{+} \mu^{+}\right) P_{N}^{\mathrm{LHCb}} \simeq(1.10 \pm 0.27) \%, \\
& \epsilon_{D}^{\mathrm{LHCb}}\left(B_{s} \rightarrow D_{s}^{-} \pi^{-} \mu^{+} \mu^{+}\right) P_{N}^{\mathrm{LHCb}} \simeq(0.89 \pm 0.22) \% .
\end{aligned}
$$

With these values, the relative uncertainty on $N_{\exp }^{\mathrm{LHCb}}$ is of $32 \%$ for both LNV modes.

The LHCb experiment performance during LHC Run 1 can be found in Ref. [62]. During LHC Run 2, the expectation is to collect $10 \mathrm{fb}^{-1}$ at the LHC nominal construction energy of a center of mass of $14 \mathrm{TeV}$. Already some work has been developed for the future $\mathrm{LHCb}$ upgrade, LHC Run 3, for which integrated luminosity of the order of $50 \mathrm{fb}^{-1}$ is expected. Assuming the above assumptions on efficiency and cross section, Fig. 1 shows the number of expected events to be observed in the $\mathrm{LHCb}$ experiment as a function of branching fraction for $|\Delta L|=2$ modes of $B_{s}$ meson. The figure shows red and magenta functions, corresponding to LHC Run 2 and LHC Run 3, respectively. Table III shows the expected signal events at the LHCb experiment for some selected values of the branching ratio, given LHC Run 2 and LHC Run 3 expected integrated luminosities. We can see that values of the branching fractions of the order $\mathcal{O}\left(10^{-9}-10^{-8}\right)$ for $B_{s}^{0} \rightarrow K^{-} \pi^{-} \mu^{+} \mu^{+}$and $\mathcal{O}\left(10^{-8}-10^{-7}\right)$ for $B_{s}^{0} \rightarrow D_{s}^{-} \pi^{-} \mu^{+} \mu^{+}$ might be within the experimental sensitivity of the LHCb.

\section{B. CMS experiment}

We also consider the possible sensitivity of the CMS experiment to the LNV signals from $B_{s}$ meson decays. The expected number of event for the CMS experiment is written as

$$
\begin{aligned}
N_{\exp }^{\mathrm{CMS}}=\sigma & \left(p p \rightarrow B_{s} X\right) \mathrm{BR}\left(B_{s} \rightarrow \Delta L=2\right) \\
& \times \epsilon_{D}^{\mathrm{CMS}}\left(B_{s} \rightarrow \Delta L=2\right) P_{N}^{\mathrm{CMS}} \mathcal{L}_{\mathrm{int}}^{\mathrm{CMS}},
\end{aligned}
$$

where $\mathcal{L}_{\text {int }}^{\text {CMS }}$ is the integrated luminosity recorded by the CMS experiment from proton-proton collisions delivered by the LHC; $\sigma\left(p p \rightarrow B_{s} X\right)$ is the $B_{s}$ meson production cross section in the CMS experiment acceptance; $\epsilon_{D}^{\mathrm{CMS}}\left(B_{s} \rightarrow \Delta L=2\right)$ is the efficiency to reconstruct and identify the signal events, which includes the trigger efficiency; $P_{N}^{\mathrm{CMS}}$ is a factor that accounts for the CMS experiment acceptance to the decay of the neutrino; and $\operatorname{BR}\left(B_{s} \rightarrow \Delta L=2\right)$ is the $B_{s}$ meson branching fraction.

The CMS experiment acceptance to the signal depends on its tracker capabilities to reconstruct charged particles, especially pions and muons. Muons are reconstructed using the tracking system and the muon chambers, while pions are reconstructed by the tracker solely. The decay products from the $B_{s}$ are not very energetic. For this study, we 

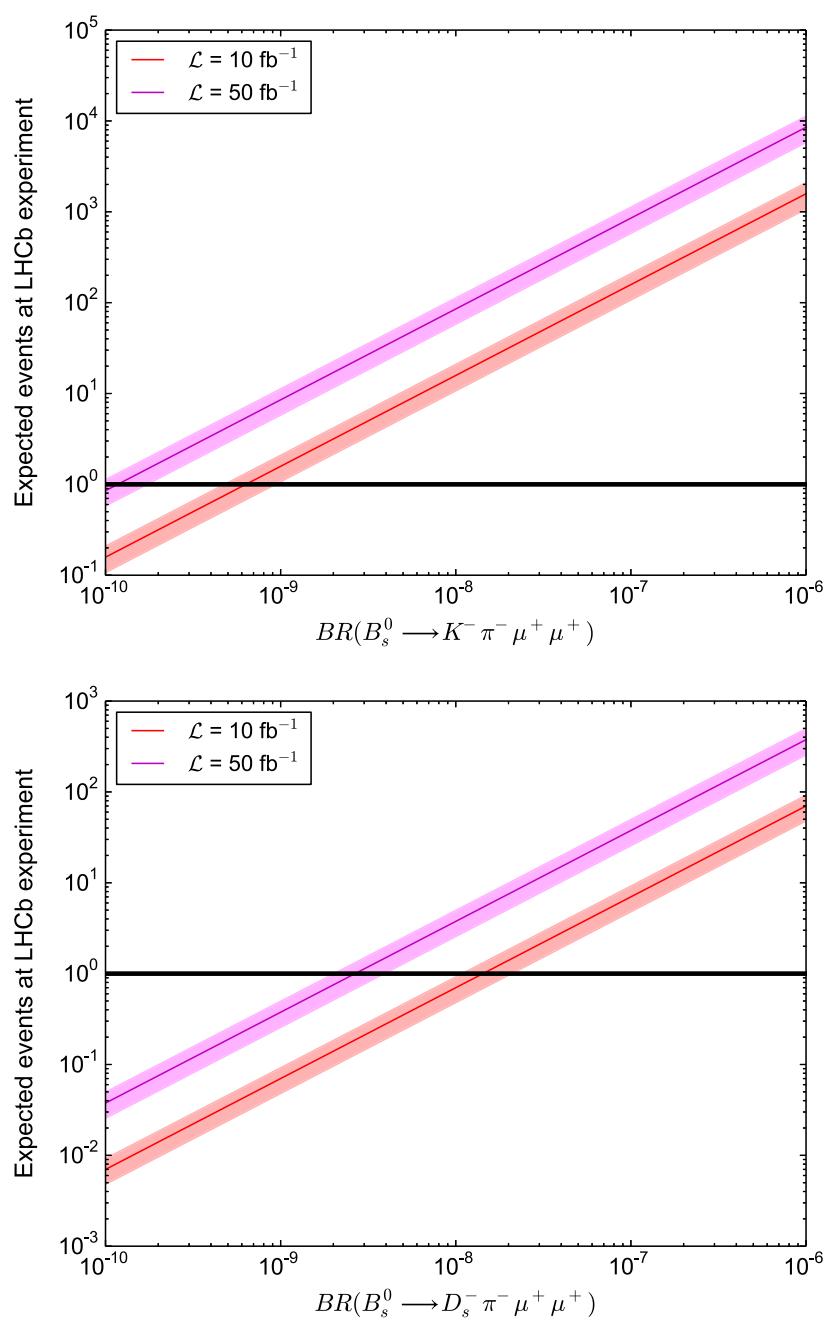

FIG. 1. Number of expected events of the process $B_{s}^{0} \rightarrow$ $K^{-} \pi^{-} \mu^{+} \mu^{+}$(top) and $B_{s}^{0} \rightarrow D_{s}^{-} \pi^{-} \mu^{+} \mu^{+}$(bottom) to be observed in the $\mathrm{LHCb}$ experiment as a function of their branching fractions for a luminosity of $10 \mathrm{fb}^{-1}$ (red) and $50 \mathrm{fb}^{-1}$ (magenta). The solid black line shows the central value, while the filled area shows the $1-\sigma$ uncertainty.

consider that the muons and pions from signal events have a $p_{T}<20 \mathrm{GeV}$. The CMS experiment has shown to be $90 \%$ efficient in reconstructing charged tracks in the mentioned $p_{T}$ range [63]. However, these studies were performed for a center-of-mass energy of $7 \mathrm{TeV}$; we consider that these results also stand for $13 \mathrm{TeV}$. In addition, we also assume that the reconstruction efficiency of muons is $90 \%$, following the results from Ref. [64].

We use the same techniques as in Ref. [47] to make a rough estimate of the CMS experiment efficiency to reconstruct the signal events. From some analyses performed with the CMS experiment for similar events $[65,66]$, we can assume that the efficiency for the events from $B_{s}^{0} \rightarrow K^{-} \pi^{-} \mu^{+} \mu^{+}$will be approximately the same $(1.56 \pm 0.05) \%$. For the decay channel $B_{s}^{0} \rightarrow D_{s}^{-} \pi^{-} \mu^{+} \mu^{+}$, we need to consider the further decay of the $D_{s}^{-}$meson.
TABLE III. Number of expected events at the LHCb for some selected values of the branching ratio (BR) of $B_{s}^{0} \rightarrow K^{-} \pi^{-} \mu^{+} \mu^{+}$ and $B_{s}^{0} \rightarrow D_{s}^{-} \pi^{-} \mu^{+} \mu^{+}$.

\begin{tabular}{lccc}
\hline \hline Mode & $\mathcal{L}_{\text {int }}^{\mathrm{LHCb}}\left(\mathrm{fb}^{-1}\right)$ & $\mathrm{BR}$ & $N_{\exp }^{\mathrm{LHCb}}$ \\
\hline$B_{s}^{0} \rightarrow K^{-} \pi^{-} \mu^{+} \mu^{+}$ & 50 & $10^{-6}$ & $8522 \pm 2727$ \\
& & $10^{-7}$ & $852 \pm 273$ \\
& & $10^{-8}$ & $85 \pm 27$ \\
& $10^{-9}$ & $9 \pm 3$ \\
& 10 & $10^{-6}$ & $1583 \pm 506$ \\
& & $10^{-7}$ & $158 \pm 51$ \\
& & $10^{-8}$ & $16 \pm 5$ \\
$B_{s}^{0} \rightarrow D_{s}^{-} \pi^{-} \mu^{+} \mu^{+}$ & & $10^{-9}$ & $2 \pm 1$ \\
& & $10^{-6}$ & $376 \pm 120$ \\
& & $10^{-7}$ & $37 \pm 12$ \\
& & $10^{-8}$ & $4 \pm 1$ \\
& & $10^{-6}$ & $70 \pm 22$ \\
\hline \hline
\end{tabular}

With the CMS experiment, it is not possible to distinguish from a charged track left in the detector by a pion or a kaon. Therefore, we consider all the possible decays of $D_{s}^{-}$into three charged tracks. Considering world averages for $K \pi \pi$ or $K K \pi$ decay branching fractions [2], we can derive that the $\operatorname{BR}\left(D_{s}^{-} \rightarrow 3\right.$ charged tracks $)=13.00 \pm 1.96$. Taking into account this additional branching fraction and the fact that we need to identify two additional charged tracks, we can plug an additional $90 \%$ efficiency factor for the track to obtain the total efficiency for the $B_{s}^{0} \rightarrow D_{s}^{-} \pi^{-} \mu^{+} \mu^{+}$ channel. We obtain that $\epsilon_{D}^{\mathrm{CMS}}\left(B_{s}^{0} \rightarrow D_{s}^{-} \pi^{-} \mu^{+} \mu^{+}\right)=$ $(1.26 \pm 0.04) \%$.

Considering the distance the neutrino can fly in the detector, we restrict the discussion to lifetimes between $\tau_{N}=1$ and $1000 \mathrm{ps}$, where the detector has sensitivity. The neutrino originates from the decay of $B_{s}$. The mean lifetime of $B_{s}$ meson is $1.505 \mathrm{ps}$ [2]. Considering that the mean momentum of $B_{s}$ is $20 \mathrm{GeV}$, from Table 1 in Ref. [65], the Lorentz time dilation factor for $B_{s}$ is $\frac{p}{M} \approx 4$, implying a decay length of $0.2 \mathrm{~cm}$. For the neutrino, we consider that $\frac{p}{M} \approx 1$ as it proceeds from the $B_{s}$ decay. Therefore, the total decay length of the neutrino, taking into account the initial decay length of $B_{s}$, is $L_{N}=0.2 \mathrm{~cm}(30.2 \mathrm{~cm})$ for $\tau_{N}=$ $1 \mathrm{ps}$ (1000 ps) lifetime. Accordingly, with the studies performed in Ref. [63], the reconstruction efficiency in the tracker is degraded in terms of the distance in the tracker system from where the traces originate. From the same study, the reconstruction efficiency of tracks originating at $30 \mathrm{~cm}$ from the collision point is $55 \%$, while for just $1 \mathrm{~cm}$, it is $100 \%$. The relative uncertainty applied on the overall reconstruction efficiency from CMS results is $18 \%$. It can be expected that differences from these assumptions would be found if a full study were done using the most recent energies used by the LHC. However, we expect to cover these differences by the uncertainty assigned. 
Additionally, the cross section of the $B_{s}^{0}$ meson production from proton-proton collisions in the geometrical acceptance of the CMS experiment is obtained from Ref. [65]. The $\sigma\left(p p \rightarrow B_{s}^{0}\right) \times \mathrm{BR}\left(B_{s}^{0} \rightarrow J / \psi \phi\right)=$ $6.9 \pm 0.6 \pm 0.6 \mathrm{nb}$ at $7 \mathrm{TeV}$, and taking $\operatorname{BR}\left(B_{s}^{0} \rightarrow J / \psi \phi\right)=$ $(1.07 \pm 0.08) \times 10^{-3}$, the pure production cross section for proton-proton collisions at $7 \mathrm{TeV}$ is $\sigma\left(p p \rightarrow B_{s}^{0}\right)=$ $(6.45 \pm 0.09) \times 10^{3} \mathrm{nb}$. Thus, assuming that the cross section increases as the center-of-mass energy, the $B_{s}^{0}$ production cross section at $13 \mathrm{TeV}$ proton-proton collisions is $\sigma\left(p p \rightarrow B_{s}^{0}\right)=(11.98 \pm 0.17) \times 10^{3} \mathrm{nb}$.

Figure 2 shows the results for the expected number of events in the CMS experiment, using the above estimations. Three benchmark luminosities are used: $\mathcal{L}_{\text {int }}^{\mathrm{CMS}}=30,300$, and $3000 \mathrm{fb}^{-1}$. Table IV is used to quote explicitly some of the results obtained. We observe that for 30 and $300 \mathrm{fb}^{-1}$
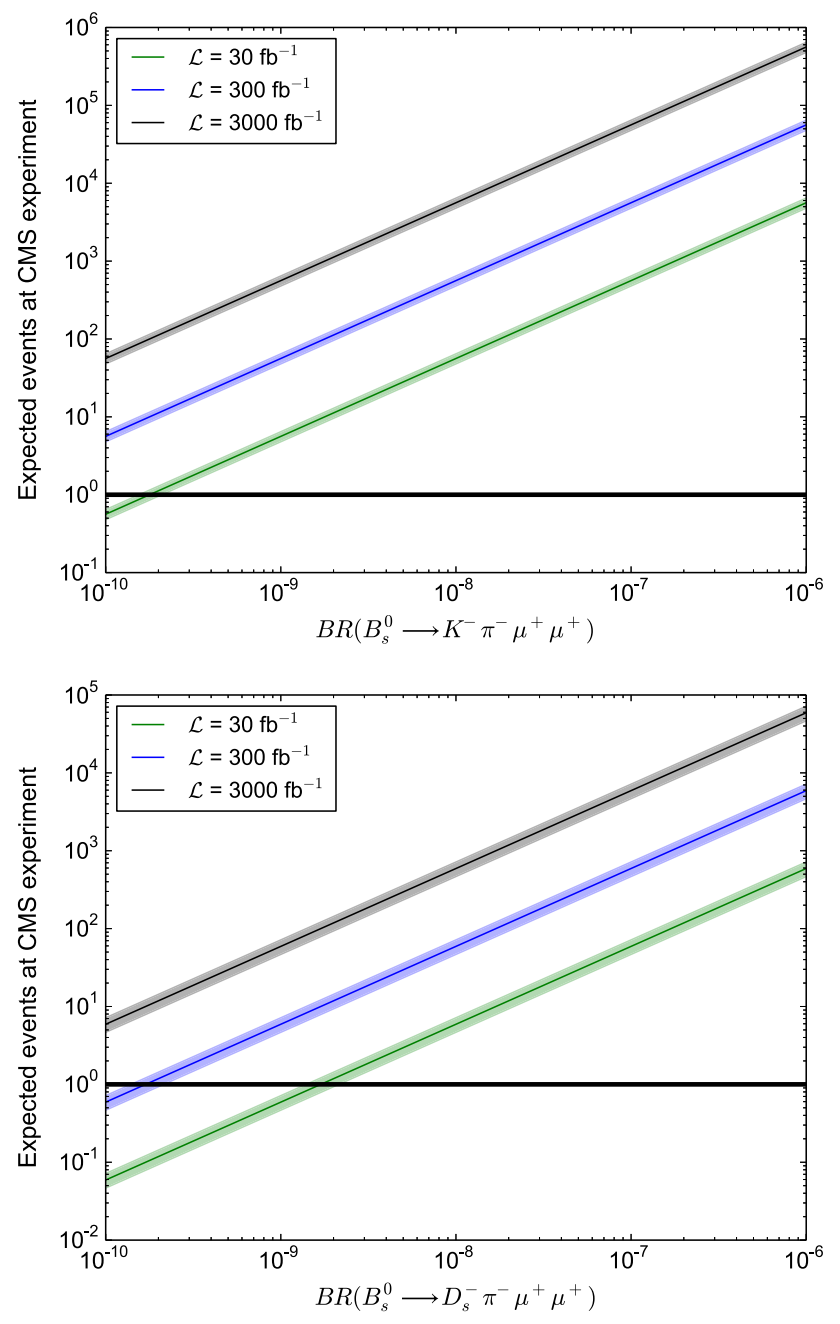

FIG. 2. Expected events in the CMS experiment for $B_{s}^{0} \rightarrow$ $K^{-} \pi^{-} \mu^{+} \mu^{+}$(top) and $B_{s}^{0} \rightarrow D_{s}^{-} \pi^{-} \mu^{+} \mu^{+}$(bottom) as a function of the branching fraction of the final state considered and for three benchmark luminosities: 30 (green), 300 (blue), and 3000 (gray) $\mathrm{fb}^{-1}$. The central value is shown with a solid line. The shaded area represents the associated uncertainty in a 1- $\sigma$ window.
TABLE IV. Expected number of events for the CMS experiment with three branching fractions of $10^{-6}, 10^{-7}$, and $10^{-8}$ for $B_{s}^{0} \rightarrow K^{-} \pi^{-} \mu^{+} \mu^{+}$and $B_{s}^{0} \rightarrow D_{s}^{-} \pi^{-} \mu^{+} \mu^{+}$.

\begin{tabular}{lccc}
\hline \hline Mode & $\mathcal{L}_{\text {int }}^{\mathrm{CMS}}\left(\mathrm{fb}^{-1}\right)$ & BR & $N_{\exp }^{\mathrm{CMS}}$ \\
\hline$B_{s}^{0} \rightarrow K^{-} \pi^{-} \mu^{+} \mu^{+}$ & 30 & $10^{-6}$ & $5616 \pm 825$ \\
& & $10^{-7}$ & $562 \pm 82$ \\
& & $10^{-8}$ & $56 \pm 8$ \\
& 300 & $10^{-8}$ & $562 \pm 82$ \\
$B_{s}^{0} \rightarrow D_{s}^{-} \pi^{-} \mu^{+} \mu^{+}$ & 30 & $10^{-9}$ & $56 \pm 8$ \\
& & $10^{-6}$ & $591 \pm 87$ \\
& & $10^{-7}$ & $59 \pm 9$ \\
& 300 & $10^{-8}$ & $6 \pm 1$ \\
& & $10^{-7}$ & $591 \pm 87$ \\
& & $10^{-8}$ & $59 \pm 9$ \\
\hline \hline
\end{tabular}

the CMS experiment has sensitivity to branching fractions of the order $\mathcal{O}\left(10^{-9}-10^{-8}\right)$ for $B_{s}^{0} \rightarrow K^{-} \pi^{-} \mu^{+} \mu^{+}$and $\mathcal{O}\left(10^{-8}-10^{-7}\right)$ for $B_{s}^{0} \rightarrow D_{s}^{-} \pi^{-} \mu^{+} \mu^{+}$. Such a sensitivity is very similar to the one that can be reached by the $\mathrm{LHCb}$ (see Sec. III A). We will consider these values of branching fractions as the most conservative ones to derive limits over the parameters of the heavy sterile neutrino in the next section.

\section{BOUNDS ON THE PARAMETER SPACE $\left(\boldsymbol{m}_{N},\left|\boldsymbol{V}_{\mu N}\right|^{2}\right)$}

The experimental nonobservation of $|\Delta L|=2$ processes can be reinterpreted as bounds on the parameter space of a heavy sterile neutrino $\left(m_{N},\left|V_{\mu N}\right|^{2}\right)$, namely, the squared mixing element $\left|V_{\mu N}\right|^{2}$ as a function of the mass $m_{N}$ $[10,15,20]$. Based on the analysis presented in Sec. III, here, we explore the constraints on the $\left(m_{N},\left|V_{\mu N}\right|^{2}\right)$ plane that can be achieved from the experimental searches on $B_{s}^{0} \rightarrow\left(K^{-}, D_{s}^{-}\right) \pi^{-} \mu^{+} \mu^{+}$at the LHC, namely, the LHCb and CMS experiments.

From Eq. (1), it is straightforward to obtain the relation

$$
\left|V_{\mu N}\right|^{2}=\left[\frac{\hbar \mathrm{BR}\left(B_{s}^{0} \rightarrow P^{-} \pi^{-} \mu^{+} \mu^{+}\right)}{\overline{\operatorname{BR}}\left(B_{s}^{0} \rightarrow P^{-} \mu^{+} N\right) \times \bar{\Gamma}\left(N \rightarrow \mu^{+} \pi^{-}\right) \tau_{N}}\right]^{1 / 2},
$$

where $\overline{\mathrm{BR}}\left(B_{s}^{0} \rightarrow P^{-} \mu^{+} N\right)$ and $\bar{\Gamma}\left(N \rightarrow \mu^{+} \pi^{-}\right)$are given by Eqs. (3) and (7), respectively. As was already discussed in Sec. III and following the analysis of NA48/2 [38] and $\mathrm{LHCb}$ [40], we will consider heavy neutrino lifetimes of $\tau_{N}=[1,100,1000]$ ps as benchmark points in our analysis. This will allow us to extract limits on $\left|V_{\mu N}\right|^{2}$ without any additional assumption on the relative size of the mixing matrix elements. 


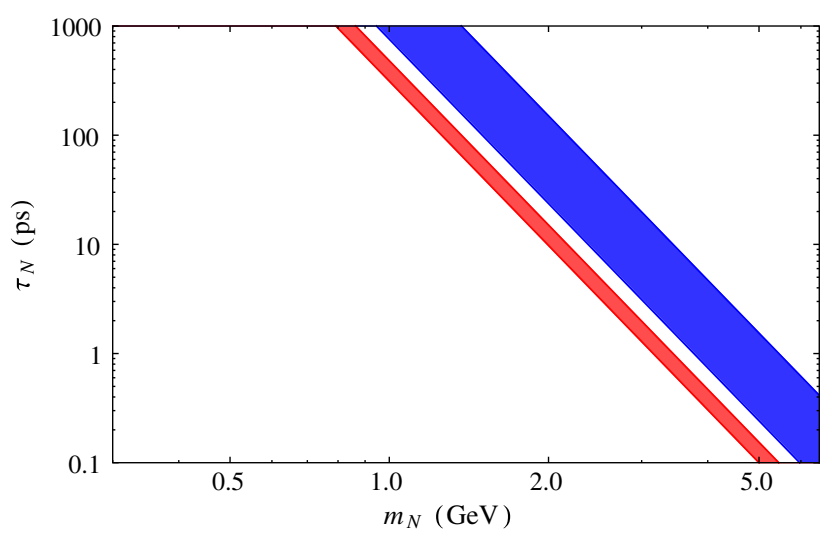

FIG. 3. Heavy neutrino lifetime $\tau_{N}$ as a function of $m_{N}$. The blue and red bands correspond to the allowed parameter spaces for $\left|V_{\tau N}\right|^{2}=10^{-3}$ and $10^{-2}$, respectively, while $\left|V_{e N}\right|^{2}$ and $\left|V_{\mu N}\right|^{2}$ vary within the range $\left[10^{-7}, 10^{-3}\right]$.

From the theoretical point of view, is worth it to justify heavy neutrino lifetimes within the domain $1 \mathrm{ps} \leq$ $\tau_{N} \leq 1000 \mathrm{ps}$ accessible to the LHCb and CMS experiments (see Secs. III A and III B). For that purpose, we will use the approximate expression for the neutrino decay width

$$
\Gamma_{N}=\frac{G_{F} m_{N}^{5}}{96 \pi^{3}}\left[8\left(\left|V_{e N}\right|^{2}+\left|V_{\mu N}\right|^{2}\right)+3\left|V_{\tau N}\right|^{2}\right],
$$

which has been previously considered in the literature $[34,35,37]$ for neutrino masses relevant to the $B_{s}$ meson decays under consideration. By considering the current bounds on $\left|V_{\ell N}\right|^{2}(\ell=e, \mu, \tau)$ given in Ref. [10], we will vary $\left|V_{e N}\right|^{2}$ and $\left|V_{\mu N}\right|^{2}$ within the range $\left[10^{-7}, 10^{-3}\right]$ and $\left|V_{\tau N}\right|^{2}$ from $10^{-3}$ to $10^{-2}$. In Fig. 3, we plot the heavy neutrino lifetime $\tau_{N}=\hbar / \Gamma_{N}$ as a function of $m_{N}$. The blue and red bands correspond to the allowed parameter spaces for $\left|V_{\tau N}\right|^{2}=10^{-3}$ and $10^{-2}$, respectively. According to Fig. 3, it is possible to obtain masses at the $\mathrm{GeV}$ scale within the lifetime domains accessible to the LHCb and CMS experiments.

In Figs. 4(a) and 4(b), we show the exclusions regions on $\left|V_{\mu N}\right|^{2}$ as a function of $m_{N}$ obtained by taking an expected sensitivity on the branching fractions of the orders $\operatorname{BR}\left(B_{s}^{0} \rightarrow K^{-} \pi^{-} \mu^{+} \mu^{+}\right)<10^{-8}$ and $<10^{-9}$, respectively. In both scenarios, the black, blue, and gray regions represent the bounds obtained for heavy neutrino lifetimes of $\tau_{N}=1,100,1000 \mathrm{ps}$, respectively. We also plot the exclusion limits obtained from searches on $|\Delta L|=2$ channels, $K^{-} \rightarrow \pi^{+} \mu^{-} \mu^{-}(\mathrm{NA} 48 / 2) \quad$ [38] and $B^{-} \rightarrow$ $\pi^{+} \mu^{-} \mu^{-}$(LHCb) [43], for comparison. For the $B^{-} \rightarrow$ $\pi^{+} \mu^{-} \mu^{-}$channel, we compare with the revised limit [32] from the LHCb analysis [43]. The limit from the $K^{-} \rightarrow$ $\pi^{+} \mu^{-} \mu^{-}$channel is taken for $\tau_{N}=1000$ ps [38]. We can observe that the most restrictive constraint is given by
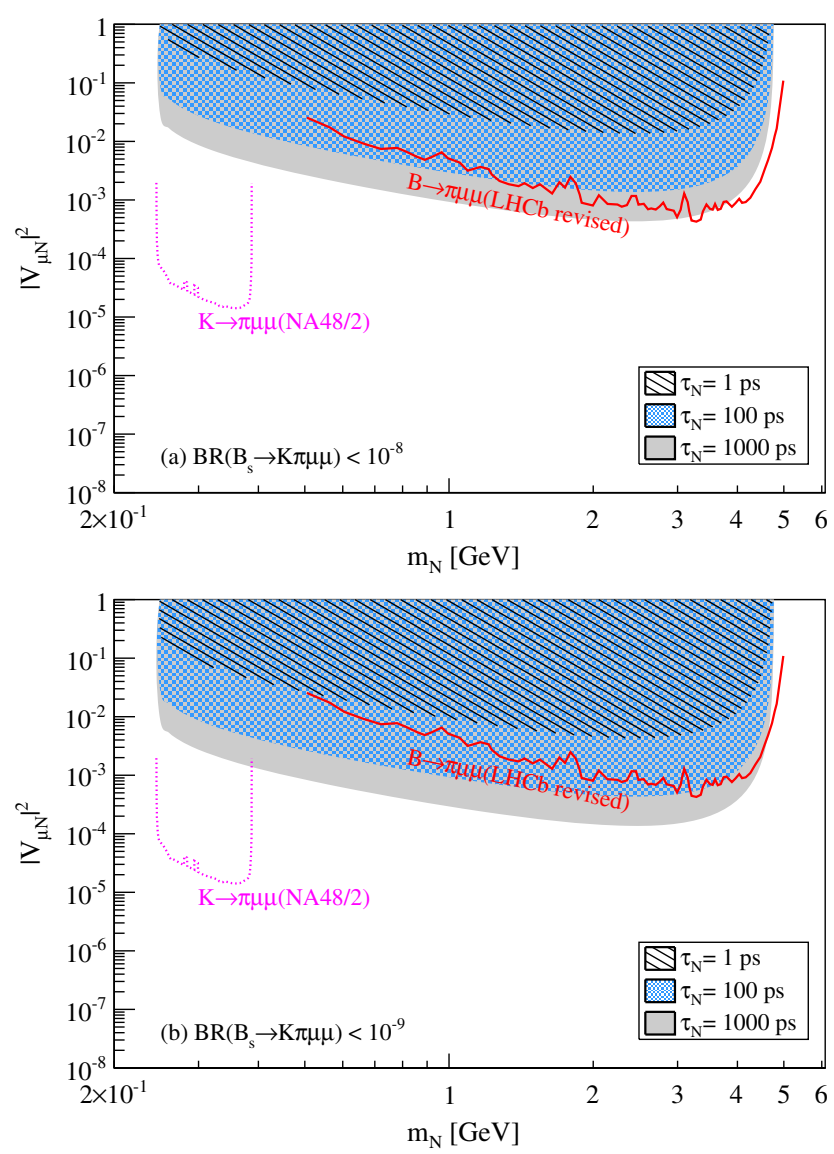

FIG. 4. Exclusion regions on the $\left(m_{N},\left|V_{\mu N}\right|^{2}\right)$ plane for (a) $\operatorname{BR}\left(B_{s}^{0} \rightarrow K^{-} \pi^{-} \mu^{+} \mu^{+}\right)<10^{-8}$ and (b) $\operatorname{BR}\left(B_{s}^{0} \rightarrow K^{-} \pi^{-} \mu^{+} \mu^{+}\right)<$ $10^{-9}$. The black, blue, and gray regions represent the bounds obtained for heavy neutrino lifetimes of $\tau_{N}=1,100,1000 \mathrm{ps}$, respectively. Limits provided by $K^{-} \rightarrow \pi^{+} \mu^{-} \mu^{-}$[38] and $B^{-} \rightarrow$ $\pi^{+} \mu^{-} \mu^{-}$[32] are also included for comparison.

$K^{-} \rightarrow \pi^{+} \mu^{-} \mu^{-}$, which can reach $\left|V_{\mu N}\right|^{2} \sim \mathcal{O}\left(10^{-5}\right)$, but only for a very narrow mass window of $[0.25,0.38] \mathrm{GeV}$. For $m_{N}>0.38 \mathrm{GeV}$, the CKM-suppressed four-body channel $B_{s}^{0} \rightarrow K^{-} \pi^{-} \mu^{+} \mu^{+}$would complement the region of $\left|V_{\mu N}\right|^{2}$ covered by the channel $B^{-} \rightarrow \pi^{+} \mu^{-} \mu^{-}$(also CKM suppressed).

For searches on $B_{s}^{0} \rightarrow D_{s}^{-} \pi^{-} \mu^{+} \mu^{+}$, in Figs. 5(a) and 5(b), we plot the exclusion curves on the $\left(m_{N},\left|V_{\mu N}\right|^{2}\right)$ plane for expected sensitivities at the $\operatorname{LHC}$ of $\operatorname{BR}\left(B_{s}^{0} \rightarrow\right.$ $\left.D_{s}^{-} \pi^{-} \mu^{+} \mu^{+}\right)<10^{-7}$ and $<10^{-8}$, respectively. Again, the black, blue, and gray regions represent the constraints obtained for heavy neutrino lifetimes of $\tau_{N}=1,100$, $1000 \mathrm{ps}$, respectively. For Majorana neutrino masses larger than $0.38 \mathrm{GeV}$, the $B_{s}^{0} \rightarrow D_{s}^{-} \pi^{-} \mu^{+} \mu^{+}$channel (CKM allowed) would be able to exclude a slightly wider region of $\left|V_{\mu N}\right|^{2}$ than $B^{-} \rightarrow \pi^{+} \mu^{-} \mu^{-}$. The reason for this is the nonsuppression for the CKM elements involved.

Additionally, in Fig. 6, we show the exclusion bounds on the parameter space $\left(m_{N},\left|V_{\mu N}\right|^{2}\right)$ coming from the 

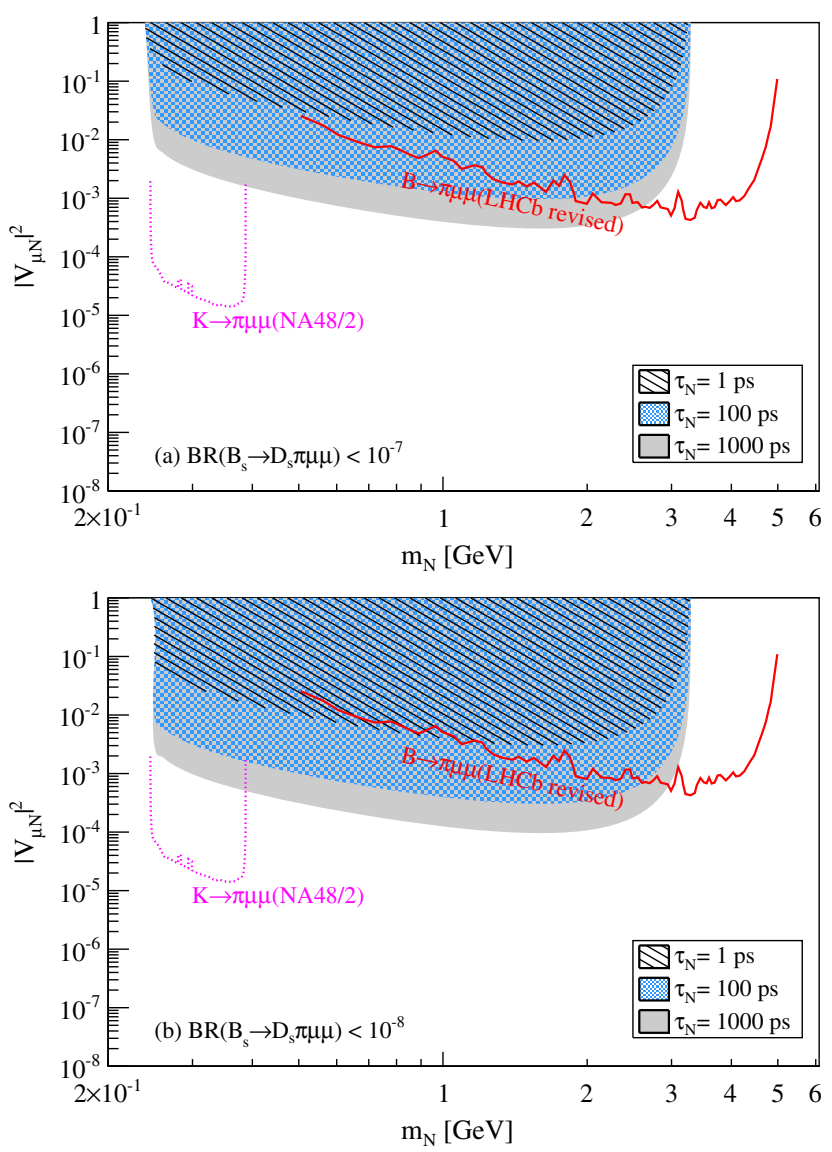

FIG. 5. The same caption as in Fig. 4 but for (a) $\operatorname{BR}\left(B_{s}^{0} \rightarrow\right.$ $\left.D_{s}^{-} \pi^{-} \mu^{+} \mu^{+}\right)<10^{-7}$ and (b) $\operatorname{BR}\left(B_{s}^{0} \rightarrow D_{s}^{-} \pi^{-} \mu^{+} \mu^{+}\right)<10^{-8}$.

Belle [67], DELPHI [68], NA3 [69], CHARMII [70], and NuTeV [71] experiments, in the mass range $[0.5,5.0] \mathrm{GeV}^{2}$ In comparison, the constraints obtained from the searches on $B_{s}^{0} \rightarrow\left(K^{-}, D_{s}^{-}\right) \pi^{-} \mu^{+} \mu^{+}$are represented by the gray and black regions, for branching fractions of BR $<10^{-9}$ and BR $<10^{-8}$, respectively. In both cases, a lifetime of $\tau_{N}=1000 \mathrm{ps}$ has been taken as a representative value. It is observed that our $|\Delta L|=2$ channels proposals are less restrictive than the bounds obtained from different search strategies, for instance, Belle [67] and DELPHI [68]. Nevertheless, keeping in mind that we have taken the most conservative values for the branching fractions derived in Secs. III A and III B, it is possible that branching fractions values of the order $\mathrm{BR}<10^{-10}$ might be accessible to the LHCb and CMS experiments (see Figs. 1 and 2); therefore, these $|\Delta L|=2$ channels would eventually provide complementary bounds.

\footnotetext{
${ }^{2}$ For recent reviews on the theoretical and experimental statuses of different $\mathrm{GeV}$-scale heavy neutrino search strategies, see Refs. [10,72-76] and references therein.
}

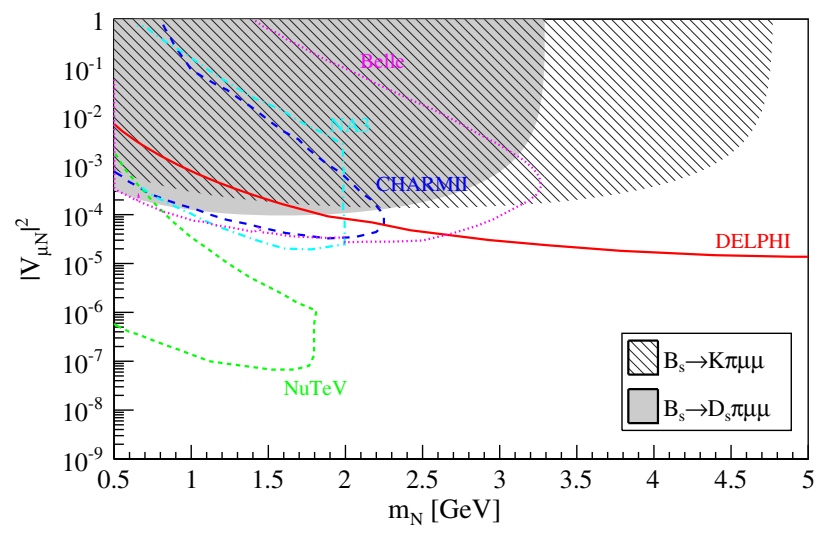

FIG. 6. Exclusion regions on the $\left(m_{N},\left|V_{\mu N}\right|^{2}\right)$ plane coming from the Belle [67], DELPHI [68], NA3 [69], CHARMII [70], and $\mathrm{NuTeV}$ [71] experiments. Limits provided by the searches on $B_{s}^{0} \rightarrow\left(K^{-}, D_{s}^{-}\right) \pi^{-} \mu^{+} \mu^{+}$are represented by the gray and black regions, respectively. See the text for details.

\section{CONCLUDING REMARKS}

We have studied the semileptonic $|\Delta L|=2$ decays of the $B_{s}$ meson $B_{s}^{0} \rightarrow P^{-} \pi^{-} \mu^{+} \mu^{+}$via the intermediate $\mathrm{GeV}$ scale on-shell Majorana neutrino $N$, namely, $B_{s}^{0} \rightarrow$ $P^{-} \mu^{+} N\left(\rightarrow \pi^{-} \mu^{+}\right)$, with $P=K, D_{s}$. To our knowledge, these LNV decays of the $B_{s}$ meson have not been investigated before from a theoretical nor from an experimental point of view. We investigated these same-sign $\mu^{+} \mu^{+}$channels and explored the sensitivity that can be reached at the LHCb and CMS experiments. We considered heavy neutrino lifetimes in the experimental (LHCb and CMS) accessible ranges of $\tau_{N}=[1,100,1000] \mathrm{ps}$, where the probability for the on-shell neutrino $N$ decay products to be inside the detector (acceptance factor $P_{N}$ ) has been taken into account in our analysis. As an outcome, it was found that for integrated luminosities collected of 10 and $50 \mathrm{fb}^{-1}$ by the LHCb experiment and 30, 300, and $3000 \mathrm{fb}^{-1}$ by the CMS experiment one would expect sensitivities on the branching fractions of the orders $\operatorname{BR}\left(B_{s}^{0} \rightarrow K^{-} \pi^{-} \mu^{+} \mu^{+}\right) \lesssim \mathcal{O}\left(10^{-9}-10^{-8}\right)$ and $\operatorname{BR}\left(B_{s}^{0} \rightarrow D_{s}^{-} \pi^{-} \mu^{+} \mu^{+}\right) \lesssim \mathcal{O}\left(10^{-8}-10^{-7}\right)$, as conservative values. For masses in the ranges $m_{N} \in[0.25,4.77] \mathrm{GeV}$ and $m_{N} \in[0.25,3.29] \mathrm{GeV}$, respectively, we extracted bounds on the parameter space $\left(m_{N},\left|V_{\mu N}\right|^{2}\right)$ that might be obtained from their experimental search. Depending on the $\tau_{N}$ value, it was found that for $m_{N}>0.38 \mathrm{GeV}$ these four-body channels may be capable of excluding a slightly wider region of $\left|V_{\mu N}\right|^{2}$ than $B^{-} \rightarrow \pi^{+} \mu^{-} \mu^{-}$(LHCb).

Consequently, the LHCb and CMS experiments have a great chance to look for heavy Majorana neutrinos in the near future, via $|\Delta L|=2$ decays of the $B_{s}$ meson. In addition, in the best-case scenario, the experimental search of these LNV channels would complement the bounds given by different search strategies (such as NA3, CHARMII, NuTeV, Belle, and DELPHI). 


\section{ACKNOWLEDGMENTS}

The work of J. Mejía-Guisao has been financially supported by Conacyt (México) under Projects No. 254409, No. 221329, and No. 250607 (Ciencia Básica) and No. 2015-2-1187 (Fronteras de la Ciencia).
N. Quintero acknowledges support from Dirección General de Investigaciones-Universidad Santiago de Cali under Project No. 935-621717-016. J. D. Ruiz-Álvarez gratefully acknowledges the support of COLCIENCIAS (Colombia).
[1] A. de Gouvêa and P. Vogel, Lepton flavor and number conservation, and physics beyond the standard model, Prog. Part. Nucl. Phys. 71, 75 (2013).

[2] C. Patrignani et al. (Particle Data Group Collaboration), Review of particle physics, Chin. Phys. C 40, 100001 (2016).

[3] See, for instance, R. N. Mohapatra et al., Theory of neutrinos: A white paper, Rep. Prog. Phys. 70, 1757 (2007); M. C. Gonzalez-Garcia and M. Maltoni, Phenomenology with massive neutrinos, Phys. Rep. 460, 1 (2008).

[4] H. Päs and W. Rodejohann, Neutrinoless double beta decay, New J. Phys. 17, 115010 (2015); W. Rodejohann, Neutrinoless double beta decay and particle physics, Int. J. Mod. Phys. E 20, 1833 (2011).

[5] S. Dell'Oro, S. Marcocci, M. Viel, and F. Vissani, Neutrinoless double beta decay: 2015 review, Adv. High Energy Phys. 2016, 2162659 (2016).

[6] J. J. Gómez-Cadenas et al., The search for neutrinoless double beta decay, Riv. Nuovo Cimento 35, 29 (2012).

[7] M. Agostini (GERDA Collaboration), Background free search for neutrinoless double beta decay with GERDA, Nature (London) 544, 47 (2017).

[8] J. B. Albert et al. (EXO-200 Collaboration), Search for Neutrinoless Double-Beta Decay with the Upgraded EXO-200 Detector, Phys. Rev. Lett. 120, 072701 (2018); Search for Majorana neutrinos with the first two years of EXO-200 data, Nature (London) 510, 229 (2014).

[9] A. Gando et al. (KamLAND-Zen Collaboration), Search for Majorana Neutrinos near the Inverted Mass Hierarchy Region with KamLAND-Zen, Phys. Rev. Lett. 117, 082503 (2016).

[10] A. Atre, T. Han, S. Pascoli, and B. Zhang, The search for heavy Majorana neutrinos, J. High Energy Phys. 05 (2009) 030 .

[11] L. S. Littenberg and R. Shrock, Upper Bounds on Lepton Number Violating Meson Decays, Phys. Rev. Lett. 68, 443 (1992); C. Dib, V. Gribanov, S. Kovalenko, and I. Schmidt, $\mathrm{K}$ meson neutrinoless double muon decay as a probe of neutrino masses and mixings, Phys. Lett. B 493, 82 (2000); L. S. Littenberg and R. Shrock, Implications of improved upper bounds on $|\Delta L|=2$ processes, Phys. Lett. B 491, 285 (2000); K. Zuber, New limits on effective Majorana neutrino masses from rare kaon decays, Phys. Lett. B 479, 33 (2000).

[12] A. Ali, A. V. Borisov, and N. B. Zamorin, Majorana neutrinos and same-sign dilepton production at $\mathrm{LHC}$ and in rare meson decays, Eur. Phys. J. C 21, 123 (2001).
[13] A. Atre, V. Barger, and T. Han, Upper bounds on leptonnumber violating processes, Phys. Rev. D 71, 113014 (2005).

[14] M. A. Ivanov and S. G. Kovalenko, Hadronic structure aspects of $K^{+} \rightarrow \pi^{-}+l_{1}^{+}+l_{2}^{+}$decays, Phys. Rev. D 71, 053004 (2005).

[15] J. C. Helo, S. Kovalenko, and I. Schmidt, Sterile neutrinos in lepton number and lepton flavor violating decays, Nucl. Phys., B853, 80 (2011).

[16] G. Cvetic, C. Dib, S. K. Kang, and C. S. Kim, Probing Majorana neutrinos in rare $K$ and $D, D_{s}, B, B_{c}$ meson decays, Phys. Rev. D 82, 053010 (2010).

[17] J. M. Zhang and G. L. Wang, Lepton-number violating decays of heavy mesons, Eur. Phys. J. C 71, 1715 (2011).

[18] S.-S. Bao, H.-L. Li, Z.-G. Si, and Y.-B. Yang, Search for Majorana neutrino signal in $B_{c}$ meson rare decay, Commun. Theor. Phys. 59, 472 (2013).

[19] Y. Wang, S.-S. Bao, Z.-H. Li, N. Zhu, and Z.-G. Si, Study Majorana neutrino contribution to $B$-meson semi-leptonic rare decays, Phys. Lett. B 736, 428 (2014).

[20] D. Milanés, N. Quintero, and C. E. Vera, Sensitivity to Majorana neutrinos in $\Delta L=2$ decays of $B_{c}$ meson at LHCb, Phys. Rev. D 93, 094026 (2016).

[21] S. Mandal and N. Sinha, Favoured $B_{c}$ decay modes to search for a Majorana neutrino, Phys. Rev. D 94, 033001 (2016).

[22] V. Gribanov, S. Kovalenko, and I. Schmidt, Sterile neutrinos in $\tau$ lepton decays, Nucl. Phys. B607, 355 (2001).

[23] D. Delepine, G. López Castro, and N. Quintero, Lepton number violation in top quark and neutral $B$ meson decays, Phys. Rev. D 84, 096011 (2011); Erratum, Phys. Rev. D86, 079905(E) (2012).

[24] G. López Castro and N. Quintero, Lepton number violation in tau lepton decays, Nucl. Phys. B, Proc. Suppl. 253-255, 12 (2014).

[25] G. López Castro and N. Quintero, Bounding resonant Majorana neutrinos from four-body $B$ and $D$ decays, Phys. Rev. D 87, 077901 (2013).

[26] H.-R. Dong, F. Feng, and H.-B. Li, Lepton number violation in $D$ meson decay, Chin. Phys. C 39, 013101 (2015).

[27] H. Yuan, T. Wang, G.-L. Wang, W.-L. Ju, and J.-M. Zhang, Lepton-number violating four-body decays of heavy mesons, J. High Energy Phys. 08 (2013) 066.

[28] G. López Castro and N. Quintero, Lepton-number-violating four-body tau lepton decays, Phys. Rev. D 85, 076006 (2012); Erratum, Phys. Rev. D86, 079904(E) (2012). 
[29] C. Dib, J. C. Helo, M. Hirsch, S. Kovalenko, and I. Schmidt, Heavy sterile neutrinos in tau decays and the MiniBooNE anomaly, Phys. Rev. D 85, 011301(R) (2012).

[30] C. Dib and C.S. Kim, Remarks on the lifetime of sterile neutrinos and the effect on detection of rare meson decays $M^{+} \rightarrow M^{\prime-} \ell^{+} \ell^{+}$, Phys. Rev. D 89, 077301 (2014).

[31] H. Yuan, Y. Jiang, T. Wang, Q. Li, and G.-L. Wang, Lepton number violating four-body tau decay, J. Phys. G 44, 115002 (2017).

[32] B. Shuve and M. E. Peskin, Revision of the LHCb limit on Majorana neutrinos, Phys. Rev. D 94, 113007 (2016).

[33] T. Asaka and H. Ishida, Lepton number violation by heavy Majorana neutrino in $B$ decays, Phys. Lett. B 763, 393 (2016).

[34] G. Cvetic and C. S. Kim, Rare decays of $B$ mesons via on-shell sterile neutrinos, Phys. Rev. D 94, 053001 (2016); Erratum, Phys. Rev. D95, 039901(E) (2017).

[35] G. Cvetic and C. S. Kim, Sensitivity limits on heavy-light mixing $\left|U_{\mu N}\right|^{2}$ from lepton number violating $B$ meson decays, Phys. Rev. D 96, 035025 (2017).

[36] G. Moreno and J. Zamora-Saa, Rare meson decays with three pairs of quasi-degenerate heavy neutrinos, Phys. Rev. D 94, 093005 (2016); J. Zamora-Saa, Resonant $C P$ violation in rare tau decay, J. High Energy Phys. 05 (2017) 110.

[37] G. Cvetic, C. Dib, C. S. Kim, and J. Zamora-Saá, Probing the Majorana neutrinos and their $C P$ violation in decays of charged scalar mesons $\pi, K, D, D_{s}, B, B_{c}$, Symmetry 7, 726 (2015); G. Cvetic, C.S. Kim, and J. Zamora-Saá, CP violation in lepton number violating semihadronic decays of $K, D, D_{s}, B, B_{c}$, Phys. Rev. D 89, 093012 (2014); $C P$ violation in $\pi^{ \pm}$meson decay, J. Phys. G 41, 075004 (2014); C. Dib, M. Campos, and C.S. Kim, CP Violation with Majorana neutrinos in $K$ Meson Decays, J. High Energy Phys. 02 (2015) 108.

[38] J. R. Batley et al. (NA48/2 Collaboration), Searches for lepton number violation and resonances in $K^{ \pm} \rightarrow \pi \mu \mu$ decays, Phys. Lett. B 769, 67 (2017).

[39] J. P. Lees et al. (BABAR Collaboration), Searches for rare or forbidden semileptonic charm decays, Phys. Rev. D 84, 072006 (2011); Search for lepton-number violating processes in $B^{+} \rightarrow h^{-} l^{+} l^{+}$decays, Phys. Rev. D 85, 071103(R) (2012).

[40] J. P. Lees et al. (BABAR Collaboration), Search for leptonnumber violating $B^{+} \rightarrow X^{-} \ell^{+} \ell^{\prime+}$ decays, Phys. Rev. D 89, 011102(R) (2014).

[41] R. Aaij et al. (LHCb Collaboration), Search for the Lepton Number Violating Decays $B^{+} \rightarrow \pi^{-} \mu^{+} \mu^{+}$and $B^{+} \rightarrow K^{-} \mu^{+} \mu^{+}$, Phys. Rev. Lett. 108, 101601 (2012); Searches for Majorana neutrinos in $B^{-}$decays, Phys. Rev. D 85, 112004 (2012).

[42] R. Aaij et al. (LHCb Collaboration), Search for $D_{(s)}^{+} \rightarrow$ $\pi^{+} \mu^{+} \mu^{-}$and $D_{(s)}^{+} \rightarrow \pi^{-} \mu^{+} \mu^{+}$decays, Phys. Lett. B 724, 203 (2013).

[43] R. Aaij et al. (LHCb Collaboration), Search for Majorana Neutrinos in $B^{-} \rightarrow \pi^{+} \mu^{-} \mu^{-}$Decays, Phys. Rev. Lett. 112, 131802 (2014).

[44] O. Seon et al. (Belle Collaboration), Search for leptonnumber-violating $B^{+} \rightarrow D^{-} \ell^{+} \ell^{\prime+}$ decays, Phys. Rev. D 84, 071106(R) (2011).
[45] Y. Miyazaki et al. (Belle Collaboration), Search for leptonflavor and lepton-number-violating $\tau \rightarrow \ell h h^{\prime}$ decay modes, Phys. Lett. B 719, 346 (2013).

[46] E. Aitala et al. (E791 Collaboration), Search for rare and forbidden charm meson decays $D^{0} \rightarrow V \ell^{+} \ell^{-}$and $h h \ell \ell$, Phys. Rev. Lett. 86, 3696 (2001).

[47] J. Mejía-Guisao, D. Milanés, N. Quintero, and J. D. RuizÁlvarez, Exploring GeV-scale Majorana neutrinos in leptonnumber-violating $\Lambda_{b}^{0}$ baryon decays, Phys. Rev. D 96, 015039 (2017).

[48] A. Faessler, M. González, S. Kovalenko, and F. Šimkovic, Arbitrary mass Majorana neutrinos in neutrinoless double beta decay, Phys. Rev. D 90, 096010 (2014); P. Benes, A. Faessler, F. Simkovic, and S. Kovalenko, Sterile neutrinos in neutrinoless double beta decay, Phys. Rev. D 71, 077901 (2005).

[49] T. Asaka, S. Blanchet, and M. Shaposhnikov, The $\nu$ MSM, dark matter and neutrino masses, Phys. Lett. B 631, 151 (2005); T. Asaka and M. Shaposhnikov, The $\nu$ MSM, dark matter and baryon asymmetry of the universe, Phys. Lett. B 620, 17 (2005).

[50] L. Canetti, M. Drewes, and M. Shaposhnikov, Sterile Neutrinos as the Origin of Dark and Baryonic Matter, Phys. Rev. Lett. 110, 061801 (2013); L. Canetti, M. Drewes, T. Frossard, and M. Shaposhnikov, Dark matter, baryogenesis and neutrino oscillations from right handed neutrinos, Phys. Rev. D 87, 093006 (2013).

[51] L. Canetti, M. Drewes, and B. Garbrecht, Probing leptogenesis with $\mathrm{GeV}$-scale sterile neutrinos at $\mathrm{LHCb}$ and Belle II, Phys. Rev. D 90, 125005 (2014).

[52] M. Drewes, B. Garbrecht, D. Gueter, and J. Klaric, Testing the low scale seesaw and leptogenesis, J. High Energy Phys. 08 (2017) 018; P. Hernández, M. Kekic, J. López-Pavón, J. Racker, and J. Salvado, Testable baryogenesis in seesaw models, J. High Energy Phys. 08 (2016) 157; P. Hernández, M. Kekic, J. López-Pavón, J. Racker, and N. Rius, Leptogenesis in GeV scale seesaw models, J. High Energy Phys. 10 (2015) 067; B. Shuve and I. Yavin, Baryogenesis through neutrino oscillations: A unified perspective, Phys. Rev. D 89, 075014 (2014).

[53] R. W. Rasmussen and W. Winter, Perspectives for tests of neutrino mass generation at the $\mathrm{GeV}$ scale: Experimental reach versus theoretical predictions, Phys. Rev. D 94, 073004 (2016).

[54] A. de Gouvea, GeV seesaw, accidentally small neutrino masses, and Higgs decays to neutrinos, arXiv:0706.1732.

[55] J. L. Rosner, S. Stone, and R. S. Van de Water, Leptonic decays of charged Pseudoscalar mesons-2015, arXiv: 1509.02220 .

[56] C. J. Monahan, H. Na, C. M. Bouchard, G. P. Lepage, and J. Shigemitsu (HPQCD Collaboration), $B_{s} \rightarrow D_{s} \ell \nu$ form factors and the fragmentation fraction ratio $f_{s} / f_{d}$, Phys. Rev. D 95, 114506 (2017).

[57] C. M. Bouchard, G. P. Lepage, C. Monahan, H. Na, and J. Shigemitsu, $B_{s} \rightarrow K \ell \nu$ form factors from lattice QCD, Phys. Rev. D 90, 054506 (2014).

[58] R. Aaij et al. (LHCb Collaboration), Measurement of $\sigma(p p \rightarrow b \bar{b} X)$ at $\sqrt{s}=7 \mathrm{TeV}$ in the forward region, Phys. Lett. B 694, 209 (2010). 
[59] Y. Amhis et al. (Heavy Flavor Averaging Group Collaboration), Averages of $b$-hadron, $c$-hadron, and $\tau$-lepton properties as of summer 2016, Eur. Phys. J. C 77, 895 (2017).

[60] R. Aaij et al. (LHCb Collaboration), Angular analysis and differential branching fraction of the decay $B_{s}^{0} \rightarrow \phi \mu^{+} \mu^{-}$, J. High Energy Phys. 09 (2015) 179.

[61] R. Aaij et al. (LHCb Collaboration), Search for massive long-lived particles decaying semileptonically in the $\mathrm{LHCb}$ detector, Eur. Phys. J. C 77, 224 (2017).

[62] R. Aaij et al. (LHCb Collaboration), LHCb detector performance, Int. J. Mod. Phys. A 30, 1530022 (2015).

[63] S. Chatrchyan et al. (CMS Collaboration), Description and performance of track and primary-vertex reconstruction with the CMS tracker, J. Instrum. 9, P10009 (2014).

[64] S. Chatrchyan et al. (CMS Collaboration), Report No. CERN-CMS-DP-2014-020, 2014.

[65] S. Chatrchyan et al. (CMS Collaboration), Measurement of the strange $B_{s}^{0}$ meson production cross section with $J / \psi \phi$ decays in $p p$ collisions at $\sqrt{s}=7 \mathrm{TeV}$, Phys. Rev. D 84, 052008 (2011).

[66] V. Khachatryan et al. (CMS Collaboration), Angular analysis of the decay $B^{0} \rightarrow K^{* 0} \mu^{+} \mu^{-}$from pp collisions at $\sqrt{s}=8 \mathrm{TeV}$, Phys. Lett. B 753, 424 (2016).

[67] D. Liventsev et al. (Belle Collaboration), Search for heavy neutrinos at Belle, Phys. Rev. D 87, 071102(R) (2013); Erratum, Phys. Rev. D 95, 099903(E) (2017)].
[68] P. Abreu et al. (DELPHI Collaboration), Search for neutral heavy leptons produced in $\mathrm{Z}$ decays, Z. Phys. C 74, 57 (1997).

[69] J. Badier et al. (NA3 Collaboration), Mass and lifetime limits on new longlived particles in $300 \mathrm{GeV} / \mathrm{c} \pi^{-}$interactions, Z. Phys. C 31, 21 (1986).

[70] P. Vilain et al. (CHARM II Collaboration), Search for heavy isosinglet neutrinos, Phys. Lett. B 343, 453 (1995); Search for heavy isosinglet neutrinos, Phys. Lett. B 351, 387 (1995).

[71] A. Vaitaitis et al. (NuTeV Collaboration), Search for neutral heavy leptons in a high-energy neutrino beam, Phys. Rev. Lett. 83, 4943 (1999).

[72] M. Drewes, Phenomenology of right handed neutrinos, Int. J. Mod. Phys. E 22, 1330019 (2013).

[73] M. Drewes and B. Garbrecht, Combining experimental and cosmological constraints on heavy neutrinos, Nucl. Phys. B921, 250 (2017).

[74] F. F. Deppisch, P. S. Bhupal Dev, and A. Pilaftsis, Neutrinos and collider physics, New J. Phys. 17, 075019 (2015).

[75] A. de Gouvêa and A. Kobach, Global constraints on a heavy neutrino, Phys. Rev. D 93, 033005 (2016).

[76] E. Fernandez-Martinez, J. Hernandez-Garcia, and J. LopezPavon, Global constraints on heavy neutrino mixing, J. High Energy Phys. 08 (2016) 033. 